\title{
N’Gaous (wilaya de Batna) : Géographie et Préhistoire
}

Jean-Louis Ballais

\section{OpenEdition}

1 Journals

Édition électronique

URL : https://journals.openedition.org/encyclopedieberbere/2735

DOI : 10.4000/encyclopedieberbere.2735

ISSN : 2262-7197

Éditeur

Peeters Publishers

\section{Édition imprimée}

Date de publication : 5 octobre 2012

Pagination : 5536-5538

ISBN : 978-90-429-2718-6

ISSN : 1015-7344

Référence électronique

Jean-Louis Ballais, « N'Gaous (wilaya de Batna) : Géographie et Préhistoire », Encyclopédie berbère [En ligne], 34 | 2012, document N52, mis en ligne le 15 décembre 2020, consulté le 17 février 2022. URL: http://journals.openedition.org/encyclopedieberbere/2735; DOI : https://doi.org/10.4000/ encyclopedieberbere. 2735

Ce document a été généré automatiquement le 17 février 2022.

(c) Tous droits réservés 


\title{
N'Gaous (wilaya de Batna) : Géographie et Préhistoire
}

\author{
Jean-Louis Ballais
}

1 N'Gaous est surtout connu par ses sites à industries acheuléennes, mais le site et ses environs ont été occupés à plusieurs reprises depuis des milliers d'années, en raison de l'attrait de ses puissantes exsurgences.

2 Ces sources (valeurs moyennes des débits : Tinibaouine : 143 l/s, N'Gaous : 188,5 l/s, Bou M'Guer : 132 l/s, Séfiane : 106 l/s (Bellion 1976, p. 168) drainent l'extrémité sud-ouest du massif du Bellezma quand il vient s'ennoyer sous la dépression du Hodna. Ce sont encore les descriptions de Bellion (1976) et Bellion et Magagnosc (1982) qui constituent la base des connaissances du modelé. Là, comme c'est le cas général au Maghreb, plusieurs glacis étagés ont été élaborés au cours du Quaternaire. L'originalité du site tient à l'existence de quatre générations de travertins fossiles, eux aussi étagés. Plus précisément, chaque génération de travertins s'est accumulée dans l'entaille linéaire creusée par les oueds dans la génération de glacis précédente. La stratigraphie se complique du fait que, parfois, l'accumulation du travertin (qui atteint jusqu'à $25 \mathrm{~m}$ d'épaisseur) a fini par déborder sur le glacis antérieur. La localisation des travertins est liée à celle des sources qui les ont accumulés, et qui s'est déplacée vers l'aval au cours $\mathrm{du}$ temps. Leur genèse s'est effectuée sur des périodes longues, avec des variations que les descriptions minutieuses de Bellion \& Magagnosc (1982) ont bien mis en évidence, mais toujours sur des pentes, parfois très faibles, et jamais dans des nappes d'eau lacustres, contrairement aux affirmations d'A. Amara (2007). Au cours d'une séquence morphoclimatique quaternaire, les travertins succèdent à la période d'humidité maximum et se placent donc dans une période plus aride, ce que confirme l'existence de poussières gypseuses prélevées au même moment sur la sebkha du Hodna. Cette localisation temporelle a été confirmée dans le cas des travertins du massif des Aurès, tout proche (Ballais 1981, p. 422).

3 Très induré, épais de 2 à $9 \mathrm{~m}$, le travertin $\mathrm{A}$ n'est plus représenté que par de rares buttes isolées, à une exception près. Le travertin $B$ se distingue par son épaisseur très importante (de $4 \mathrm{~m}$ jusqu'à $25 \mathrm{~m}$ ), sa couleur brun-rouille et la présence de bifaces 
interstratifiés de l'Acheuléen moyen. Le travertin C est blanc, moins induré, épais de 3 à $7 \mathrm{~m}$. Enfin, le travertin D est gris, souvent conservé sous forme de témoins au fond des vallons à la tête des oueds et sur les versants vers l'aval, tendre, avec des passées noires locales, d'épaisseur très variable et qui varie très rapidement (Bellion, Ballais et Guiraud 1987 ; Bellion et Magagnosc 1982).

Dès le début de leurs recherches sur le Kef Sefiane, au sud-est de N'Gaous, Bellion et Magagnosc ont découvert des bifaces en surface (Magagnosc in litteris), puis en place, dans le travertin $B$ attribué alors à l'Amiro-Tensiftien (Pléistocène moyen). Malheureusement, 35 ans après leur découverte, les sites acheuléens n'ont toujours pas bénéficié des fouilles et des publications qu'ils méritent. A. Amara (2007) signale l'existence de trois sites acheuléens, qu'il attribue respectivement à l'Acheuléen très archaïque, à l'Acheuléen et à l'Acheuléen évolué, sur la base de rares artefacts en calcaire, calcaire siliceux et silex (respectivement 28, 29 et 4).

5 La richesse en eau de N'Gaous et de ses environs a attiré les populations humaines à plusieurs reprises. D'abord, à l'Holocène inférieur, des populations épipaléolithiques, en bordure de l'oued Gouriane : là, des éclats de silex sont éparpillés dans le travertin D peu consolidé, gris ou jaunâtre, qui recouvre le glacis II. Ils sont associés à Rumina decollata et Helix melanostoma. Dans un autre site, probablement élassolithique, qui comporte une quarantaine d'artefacts, un peu plus au nord, un fragment retouché pourrait être affecté par une cupule de gel (Ballais et C. Roubet 1981-1982). Des sites capsiens reposeraient sur le glacis postérieur (Bellion et Magagnosc 1982).

6 Tout récemment (Sahed 2010), une importante nécropole protohistorique vient d'être découverte à Séfiane: plus de 300 monuments: tumulus, bazinas, aires funéraires (cromlechs). La fouille de quelques tombes a fourni très peu de matériel archéologique (poterie modelée, ossements humains) mais a permis une datation au $\mathrm{C}^{14}$ sur charbons de bois : $3209 \pm 195$ BP dans une bazina à degrés (MF nº IV).

$7 \rightarrow$ Voir aussi N53 et N55 «Nicibes/Nicives »

\section{BIBLIOGRAPHIE}

AMARA A., 2007 - « Premiers résultats de l'étude de la stratigraphie et du Paléolithique inférieur de N'Gaous, Monts des Bellezma (Aurès) », Le Paléolithique inférieur en Algérie, Cultures et Environnements, Alger, Travaux du CNRPAH, n.s., 3, p. 21-48.

BALLAIS J.-L., 1981 - Recherches géomorphologiques dans les Aurès (Algérie), Thèse doctorat ès-Lettres et Sciences Humaines, Paris I, VII+566 p.

BALLAIS J.-L., ROUBET C., 1981-1982 - « Morphogenèse et préhistoire dans les Aurès (Algérie) », Rev. Géol. dyn. et Géogr. phys., vol. 23, fasc. 5, p. 375-384.

BELLION Y., 1976 - Étude géologique et hydrogéologique de la terminaison occidentale des Monts du Bellezma (Algérie), Thèse doctorat $3^{\mathrm{e}}$ cycle, Université Paris VI, $221 \mathrm{p}$. 
BELLION Y., BALLAIS J.-L., GUIRAUD R., 1987 - Carte géologique au 1/50 000 N’Gaous, Alger, Serv. Carte géol., 1972.

BELLION Y., MAGNAGNOSC J. S., 1982 - « Les travertins du Kef Séfiane, extrémité occidentale des Monts de Batna-Bellezma (confins Hodna-Aurès ; Algérie du Nord). Position et signification dans le contexte morphogénétique régional », in J. NICoD éd., Phénomènes karstiques III, Mémoires et Documents du CNRS, p. 181-202.

LANCEL S., 2008 - L'Algérie antique, Paris, Éditions Place des Victoires, 259 p.

LAPORTE J.-P., 2006 - « N'gaous (Numidie) : deux inscriptions nouvelles », Mélanges à la mémoire d'Hans Georg Pflaum : H.-G. Pflaum, Un historien du XX siècle, Paris/Genève, EPHE/Droz, p. 89-109.

SAHED T. A., 2010 - «Contribution à l'étude de la nécropole protohistorique de Sefiane (région de N'gaous) », Antropo, 21, p. 61-77. www.didac.ehu.es/antropo

INDEX

Mots-clés : Algérie 